\title{
Anti-tumour necrosis factor treatment for the prevention of ischaemic events in patients with deficiency of adenosine deaminase 2 (DADA2)
}

Samantha Cooray ${ }^{1}$, Ebun Omyinmi ${ }^{1}$, Ying Hong ${ }^{1}$, Charalampia Papadopoulou ${ }^{1}$, Lorraine Harper ${ }^{2}$, Eslam Al-Abadi ${ }^{3}$ Ruchika Goel ${ }^{2}$, Shirish Dubey ${ }^{4}$, Mark Wood ${ }^{5}$, Stephen Jolles ${ }^{6}$, Stefan Berg ${ }^{7}$, Maria Ekelund $^{8}$, Kate Armon ${ }^{9}$, Despina Eleftheriou ${ }^{1}$, Paul A. Brogan ${ }^{1}$

${ }^{1}$ Infection, Inflammation and Rheumatology Section, University College London Great Ormond Street Institute of Child Health, London, UK;

${ }^{2}$ Institute of Clinical Sciences, Centre for Translational Inflammation Research, University of Birmingham, Birmingham, UK;

${ }^{3}$ Rheumatology Department, Birmingham Children's Hospital, Birmingham, UK;

${ }^{4}$ Rheumatology Department, Oxford University Hospitals NHS Foundation Trust, Oxford, UK;

${ }^{5}$ Paediatric Rheumatology Department, Leeds Teaching Hospitals NHS Trust, Leeds, UK;

${ }^{6}$ Immunodeficiency Centre for Wales, University Hospital of Wales, Cardiff, UK;

7 Paediatric Rheumatology, The Queen Silvia Children's Hospital and University of Gothenburg, Gothenburg, Sweden;

${ }^{8}$ Ryhov County Hospital, Jönköping, Sweden;

${ }^{9}$ Department of Paediatric Rheumatology, Addenbrooke's Hospital, Cambridge, UK

Correspondence to: Samantha Cooray, Infection, Inflammation and Rheumatology Section, University College London Great Ormond Street Institute of Child Health, 30 Guilford St, Holborn, London WC1N 1EH London, UK. E-mail: s.cooray@ucl.ac.uk. 


\section{Abstract}

Objective. To evaluate the impact of anti-Tumour Necrosis Factor- $\alpha$ (anti-TNF) treatment on the occurrence of vasculitic ischaemic events in patients with deficiency of adenosine deaminase 2 (DADA2).

Methods. A retrospective analysis of DADA2 patients referred from six centres to Great Ormond Street Hospital for Children was conducted. Ischaemic events, vasculitic disease activity, biochemical, immunological, and radiological features were compared, before and after anti-TNF treatment.

Results. A total of 31 patients with genetically confirmed DADA2 were included in the study. The median duration of active disease activity prior to anti-TNF treatment was 73 months (Inter-quartile range [IQR] 27.5-133.5 months). Twenty seven/31 patients received anti-TNF treatment for a median of 32 months (IQR 12.0-71.5 months). The median event rate of central nervous system (CNS) and non-CNS ischemic events before anti-TNF treatment was 2.37 per 100 patient-months (IQR 1.25-3.63); compared with 0.00 per 100 patient-months (IQR 0.0-0.0) post-treatment $(p<0.0001)$. Paediatric vasculitis activity score (PVAS) was also significantly reduced: median score of 20/63 (IQR 13.0-25.8/63) pre-treatment versus 2/63 (IQR 0.0-3.8/63), following anti-TNF treatment $(p<0.0001)$ with mild livedoid rash being the main persisting feature. Anti-TNF treatment was not effective for severe immunodeficiency or bone marrow failure, which required haematopoietic stem cell transplantation (HSCT).

Conclusion. Anti-TNF treatment significantly reduced the incidence of ischaemic events and other vasculitic manifestations of DADA2, but was not effective for immunodeficiency or bone marrow failure.

Key words: DADA2, anti-TNF, ischaemia, stroke, inflammation, vasculitis, PVAS

\section{Rheumatology key messages}

1. Deficiency of adenosine deaminase 2 is an autosomal recessive autoinflammatory disease associated with systemic vasculitis, immunodeficiency and bone marrow failure.

2. Anti-TNF significantly reduced vasculitic disease activity and prevented ischaemic events, but was not effective for immunodeficiency or marrow failure. 


\section{Introduction}

Deficiency of adenosine deaminase 2 (DADA2) is a rare autosomal recessive autoinflammatory disease caused by bi-allelic loss-of-function mutations in the ADA2 gene (previously CECR1) [1, 2]. Clinical features include systemic inflammation, vasculitis and ischaemic events such as early-onset lacunar and haemorrhagic stroke, peripheral vascular disease and renal infarcts [1-7]. Some individuals also suffer from immunodeficiency and haematological defects, ranging from immunoglobulin subclass deficiency and cytopaenias to bone marrow failure $[3,6,8]$.

The $A D A 2$ gene encodes an extracellular homodimeric protein, adenosine deaminase 2 (ADA2) that is primarily expressed in monocytes and macrophages [9]. Although ADA2 has a catalytic domain with structural homology to adenosine deaminase 1 (ADA1), it is a homodimer with 100-fold lower binding affinity for adenosine, suggesting a limited role for deamination at physiological concentrations [9]. ADA2 has homology to insect and mollusc growth factors and is heavily glycosylated [9]. It has been shown to bind to immune cells, suggesting a potential role in growth factor and innate immune signalling $[5,9]$.

Deficiency of ADA2 disrupts endothelial vascular integrity and impairs macrophage differentiation [2]. This results in a predominance of $M 1$ macrophages that secrete pro-inflammatory cytokines such as tumour necrosis factor (TNF), an important mediator of vasculitis. As with other vasculitides, severe vasculitis with arterial narrowing and platelet activation is thought to contribute to thrombotic occlusion, ischaemia and infarction [10].

There is no definitive treatment strategy for DADA2, although a number of immunomodulatory drugs have been tried $[1,2,6,11]$. A small number of patients with severe immunodeficiency or bone marrow failure have undergone allogenic haematopoietic stem cell transplantation (HSCT), which has proven beneficial $[12,13]$. Exogenous replacement of ADA2 has also been considered, however, treatment of patients with ADA2-containing fresh frozen plasma (FFP) has failed to show clinical benefit [11]. Anti-TNF treatments have demonstrated efficacy and safety in retrospective analyses, significantly improved quality-of-life and reduced inflammation and stroke risk [11]. The rarity of DADA2, however, combined with ethical challenges regarding use of placebo for this severe disease are major barriers to conducting a randomised controlled trial with anti-TNF agents. Therefore, the aim of this retrospective observational study was to describe the efficacy of anti-TNF for vasculitic manifestations of DADA2, with particular emphasis on central nervous system (CNS) and non-CNS ischaemic events. 


\section{Methods}

Great Ormond Street Hospital for Children NHS Foundation Trust is a quaternary referral centre for paediatric vasculitis and autoinflammation and provides diagnostic testing for DADA2 including genetic screening and serum ADA2 enzyme activity [6]. These studies were performed with full written consent from parents, and child assent, including consent to publish, with full institutional ethics committee approval (Ethics number: 08H071382; R\&D number 12RU11), as previously described [4, 6]. Retrospective patient data was gathered from hospital records and was fully anonymised. Genetic analysis and ADA2 enzyme activity were performed as previously described [6].

Patients were followed up every 1-12 months (as clinically indicated) and evaluated for features of DADA2 disease activity including, but not limited to, livedoid rash, fever, joint pain, peripheral vascular disease, and neurological features. Laboratory blood tests were taken at diagnosis and subsequent outpatient clinic visits to monitor inflammation, immunodeficiency and organ dysfunction. Prior to the initiation of ant-TNF treatment, QuantiFERON testing for Mycobacterium tuberculosis and a chest $\mathrm{x}$-ray were performed to exclude latent tuberculosis.

Ischaemic events were diagnosed based on clinical features together with brain magnetic resonance imaging/angiography (MRI/MRA); and/or other imaging modalities such as ultrasound scan, computed tomography (CT)-angiography, or selective visceral catheter arteriography, where indicated. Ischaemic events were categorised as either CNS or non-CNS (arteries supplying viscera and peripheries). Where the diagnosis of DADA2 was known prior to the occurrence of ischaemic events, baseline brain MRI/MRA was carried out, with further imaging performed on clinical suspicion of new ischaemic events.

The ischaemic event rate was calculated per 100 patient-months for each patient from the time of disease onset to the time of starting anti-TNF treatment. The rate of ischaemic events was also calculated after starting anti-TNF treatment per 100 patient-months from the treatment start date until $1^{\text {st }}$ July, 2020.

The paediatric vasculitis activity score (PVAS) was used to score vasculitic activity at first presentation and after anti-TNF treatment at most recent clinic visit [14]. The PVAS ranges from 063 , with higher scores denoting clinical vasculitic disease activity across 9 organ systems, and a score of 0 indicating absent activity. Differences in ischaemic event rate, PVAS and inflammatory markers were compared using a Wilcoxon matched-pairs signed rank test using GraphPad Prism Software version 8.4.3 and Microsoft Excel 2016. 


\section{Results}

Thirty one patients (14 females) of median age 15 years (IQR 12-21.75 years) and confirmed bi-allelic or compound heterozygous mutations in the ADA2 gene were included in the study (Supplementary Table 1). The median age of disease onset was 5 years (IQR 3-7.75 years) and included variable features from mild livedo racemosa to stroke or severe haematological abnormalities, with or without vasculitis. All $A D A 2$ mutations were previously reported in the literature, except the p.His91Arg homozygous missense mutation in patient 17 , located in the $\mathrm{N}$-terminal HN3 region involved in protein dimerisation (Supplementary Table 1). The patient presented acutely with severe cutaneous necrosis and reduced ADA2 activity with no other identifiable cause.

Prior to initiation of anti-TNF treatment, 26/31 patients had had one or more ischaemic event (Supplementary Table 1). Overall, there were 53 ischaemic events: 38 CNS (18 patients), and 15 non-CNS (9 patients) over a cumulative total of 2324 months (median time pre-anti-TNF treatment 73 months; IQR 27.5-133.5 months; Table 1 and Supplementary Table 1). Five patients had no history of ischaemic events.

Twenty-seven/31 patients were given anti-TNF treatment. Three patients had mild symptoms that did not warrant treatment; one patient had bone marrow failure and died of complications of sepsis and bowel perforation, having received a single dose of anti-TNF (Supplementary Tables 1 and 2). Following anti-TNF treatment, 4 patients experienced a single ischemic event (1 CNS, 3 non-CNS) over a cumulative total of 1325 months (median treatment time 32 months; IQR 12-71.5 months; Table 1 and Supplementary Table 2). Patient 5 presented with a cranial nerve IV palsy just prior to an adalimumab dose increment from $20 \mathrm{mg}$ to $40 \mathrm{mg}$ two weekly that resolved, with a normal brain MRI/MRA. Patient 9 experienced transient cold peripheries and paraesthesia. Patient 23 developed digital necrosis resulting in a partial amputation due to poor compliance with anti-TNF treatment. Patient 27 had ischaemic colitis associated with an incomplete response to anti-TNF, complicated by neutropenic infections.

The median rate of ischaemic events in DADA2 patients was 2.37 (IQR 1.25-3.63) before antTNF treatment versus 0.0 (IQR 0.00-0.00) per 100 patient-months after treatment $(p<0.0001$; Table 1 and Figure $1 \mathrm{~A}$ ). Patient 17 had a high ischaemic rate due to acute onset disease ( $<2$ months) and rapid diagnosis and treatment. There was also a significant reduction in disease activity as assessed by PVAS from a median of 20/63 (IQR 13-25.8) to 2/63 (IQR 0-3.8) after treatment ( $p<0.0001$; Figure 1B, Supplementary Table 1). Patient 11 had a severe chronic peripheral vasculitic ulcer that demonstrated complete healing after 4 months of anti-TNF treatment (Supplementary Figure 1). Inflammatory markers C Reactive Protein (CRP) and Erythrocyte Sedimentation Rate (ESR) were significantly reduced after anti-TNF treatment ( $p<0.0001$ for both markers; Figure $1 C \& D)$. In patient 
27, inflammatory markers remained high due to recurrent infections in the context of ongoing neutropaenia (Figure 2; Supplementary Table 3).

DADA2 features that persisted despite anti-TNF treatment were livedo racemosa (8 patients), arthralgia (4 patients), and headaches (3 patients). Fever was observed in three patients and coincided with ischaemic events post anti-TNF treatment. Three siblings displayed a unique phenotype with persistent upper motor neuron signs, including brisk reflexes and clonus, which did not improve with anti-TNF treatment and was unrelated to acute CNS events or damage.

All patients underwent brain MRI on presentation of neurological features or at baseline following diagnosis (Supplementary Table 4). Eighteen/31 patients also underwent head MRA imaging which was normal in all cases; 1 patient had mild single vessel tortuosity of uncertain clinical significance. Eight patients underwent catheter visceral arteriography as part of their initial diagnostic workup, with renal, hepatic or mesenteric artery abnormalities (stenosis, aneurysm) demonstrated in $4 / 8$.

In terms of anti-TNF agent, most received adalimumab (Supplementary Table 2). Four patients were started on infliximab, but $3 / 4$ suffered infusion reactions and were switched to adalimumab. One patient was switched from infliximab to etanercept after a treatment break. A further 4 patients were started directly on etanercept (Supplementary Table 2). No adverse reactions were reported with adalimumab or etanercept. Five patients remain on steroid therapy, 4 on methotrexate, 1 on azathioprine and 3 on mycophenolate mofetil (MMF) due to waning drug effectiveness or incomplete response with recurrence of systemic inflammation. One patient is being weaned off steroids. Ten patients who experienced non-haemorrhagic ischaemic events were started on low-dose aspirin, (6 lacunar stroke, 1 testicular infarct, 1 peripheral vascular disease leading to amputation), which was stopped in 5/10 patients at a median of 11 months (range 9-23 months) after the acute event.

Four patients presented with a severe haematological DADA2 phenotype with varying degrees of immunodeficiency (Supplementary Table 1). Patient 1 had thrombocytopaenia, neutropaenia and anaemia requiring granulocyte colony stimulating factor (G-CSF) which was still required post-anti-TNF treatment. Patients 3 and 19 had multi-lineage bone marrow failure, requiring regular G-CSF and blood products. Patient 19 underwent allogenic-HSCT (allo-HSCT) and subsequent splenectomy following which cell counts normalised and steroids, azathioprine and antiTNF agent were stopped. However, immunoglobulin replacement therapy was still required. Patient 3 died prior to planned allo-HSCT. Patient 27 had severe isolated neutropaenia with recurrent infections, requiring frequent G-CSF infusions, and is being worked up for allo-HSCT. Patient 22 had isolated severe red cell aplasia from birth, requiring monthly blood transfusions. Patients 30 and 31 
had neutropaenia which resolved in patient 31 with anti-TNF treatment but continues in patient 30 despite treatment. Both patients continue to have mild, treatment-resistant microcytic anaemia. Mild lymphopaenia, anaemia and IgM deficiency resolved in the majority of patients following antiTNF treatment; but IgA deficiency persisted.

Of the 31 DADA2 patients in this series, 3 remain well having never required treatment, 26 patients remain on anti-TNF, 1 patient required allo-HSCT (stopped anti-TNF), and 1 patient died. 


\section{Discussion}

Anti-TNF treatment significantly reduced both CNS and non-CNS ischaemic events in DADA2, with reduction in vasculitic disease activity and inflammatory markers. This observation strongly supports previous data suggesting that anti-TNF treatment significantly reduces the occurrence of stroke in DADA2 [11].

Anti-TNF agents used in our cohort were well tolerated and highly efficacious for major vasculitic features, but did not improve established end-organ damage. However, minor features such as livedo racemosa and headache frequently persisted. Regarding choice of anti-TNF agent, patients who had been initially started on infliximab commonly developed infusion reactions, requiring a switch to adalimumab or etanercept. This is a recognised complication, likely related to development of anti-drug antibodies against this human-mouse chimeric monoclonal antibody [15], although these were not specifically measured. Although less of an issue for adalimumab and etanercept, anti-drug antibodies may limit treatment effectiveness and remains an ongoing concern for these agents [15], particularly since anti-TNF treatment in DADA2 is lifelong.

Anti-TNF has no impact on severe neutropaenia or pancytopaenia in DADA2, allo-HSCT being the only definitive treatment for non-infarctive bone marrow sequelae $[6,11,12]$. Allo-HSCT has been demonstrated to resolve both vasculitic and haematological features of DADA2 [12]. In our patient cohort, a single adult patient underwent allo-HSCT with resolution of immunodeficiency and vasculitis. Allo-HSCT is not without risks, including graft rejection, graft-versus-host disease (GvHD) and infection. A potential less toxic alternative treatment for DADA2 is gene therapy, which has proved successful for other monogenic diseases including severe combined immunodeficiency caused by mutations in ADA1 (ADA-SCID) [16], and is actively being studied by our group.

In conclusion, we highlight the efficacy of anti-TNF treatment in controlling vasculitic disease activity and ischaemic events in DADA2; those with severe haematological manifestations may require alloHSCT. Gene therapy may be a curative option in the future for such cases. 


\section{Acknowledgements}

This work was supported in part by a grant from Rosetrees. SC also acknowledges support from an NIHR fellowship grant. PAB also acknowledges support from Great Ormond Street Hospital Children's Charity. All research at Great Ormond Street Hospital NHS Foundation Trust and UCL Great Ormond Street Institute of Child Health is made possible by the NIHR Great Ormond Street Hospital Biomedical Research Centre.

\section{Conflict of Interest}

The authors declare no conflict of interest.

\section{Data Availability Statement}

The data underlying this article are available in the article and in its online supplementary material.

\section{Supplementary Data}

Supplementary data are available at Rheumatology online. 


\section{References}

1. Navon Elkan P, Pierce SB, Segel R, Walsh T, Barash J, Padeh S, et al. Mutant adenosine deaminase 2 in a polyarteritis nodosa vasculopathy. N Engl J Med. 2014;370(10):921-31.

2. Zhou Q, Yang D, Ombrello AK, Zavialov AV, Toro C, Stone DL, et al. Early-onset stroke and vasculopathy associated with mutations in ADA2. N Engl J Med. 2014;370(10):911-20.

3. Caorsi R, Penco F, Grossi A, Insalaco A, Omenetti A, Alessio M, et al. ADA2 deficiency (DADA2) as an unrecognised cause of early onset polyarteritis nodosa and stroke: a multicentre national study. Ann Rheum Dis. 2017;76(10):1648-56.

4. Clarke K, Campbell C, Omoyinmi E, Hong $Y$, Al Obaidi $M$, Sebire $N$, et al. Testicular ischemia in deficiency of adenosine deaminase 2 (DADA2). Pediatr Rheumatol Online J. 2019;17(1):39.

5. Kaljas $Y$, Liu C, Skaldin M, Wu C, Zhou Q, Lu Y, et al. Human adenosine deaminases ADA1 and ADA2 bind to different subsets of immune cells. Cell Mol Life Sci. 2017;74(3):555-70.

6. Nanthapisal S, Murphy C, Omoyinmi E, Hong Y, Standing A, Berg S, et al. Deficiency of Adenosine Deaminase Type 2: A Description of Phenotype and Genotype in Fifteen Cases. Arthritis Rheumatol. 2016;68(9):2314-22.

7. Westendorp WF, Nederkoorn PJ, Aksentijevich I, Hak AE, Lichtenbelt KD, Braun KP. Unexplained early-onset lacunar stroke and inflammatory skin lesions: Consider ADA2 deficiency. Neurology. 2015;84(20):2092-3.

8. Ben-Ami T, Revel-Vilk S, Brooks R, Shaag A, Hershfield MS, Kelly SJ, et al. Extending the Clinical Phenotype of Adenosine Deaminase 2 Deficiency. J Pediatr. 2016;177:316-20.

9. Zavialov AV, X. Y, Spillmann D, Lauvau G, Zavialov AV. Structural Basis for the Growth Factor Activity of Human Adenosine Deaminase ADA2. J Biol Chem. 2010;285(16):2367-12377.

10. Brogan PA, Shah V, Brachet C, Harnden A, Mant D, Klein N, et al. Endothelial and platelet microparticles in vasculitis of the young. Arthritis Rheum. 2004;50(3):927-36.

11. Ombrello AK, Qin J, Hoffmann PM, Kumar P, Stone D, Jones A, et al. Treatment Strategies for Deficiency of Adenosine Deaminase 2. N Engl J Med. 2019;380(16):1582-4.

12. Hashem H, Kumar AR, Muller I, Babor F, Bredius R, Dalal J, et al. Hematopoietic stem cell transplantation rescues the hematological, immunological, and vascular phenotype in DADA2. Blood. 2017;130(24):2682-8.

13. Hashem H, Vatsayan A, Gupta A, Nagle K, Hershfield M, Dalal J. Successful reduced intensity hematopoietic cell transplant in a patient with deficiency of adenosine deaminase 2 . Bone Marrow Transplant. 2017;52(11):1575-6.

14. Dolezalova P, Price-Kuehne FE, Ozen S, Benseler SM, Cabral DA, Anton J, et al. Disease activity assessment in childhood vasculitis: development and preliminary validation of the Paediatric Vasculitis Activity Score (PVAS). Ann Rheum Dis. 2013;72(10):1628-33.

15. Atiqi S, Hooijberg F, Loeff FC, Rispens T, Wolbink GJ. Immunogenicity of TNF-Inhibitors. Front Immunol. 2020;11:312. 
16. Gaspar HB, Cooray S, Gilmour KC, Parsley KL, Zhang F, Adams S, et al. Hematopoietic stem cell gene therapy for adenosine deaminase-deficient severe combined immunodeficiency leads to long-term immunological recovery and metabolic correction. Sci Transl Med. 2011;3(97):97ra80. 